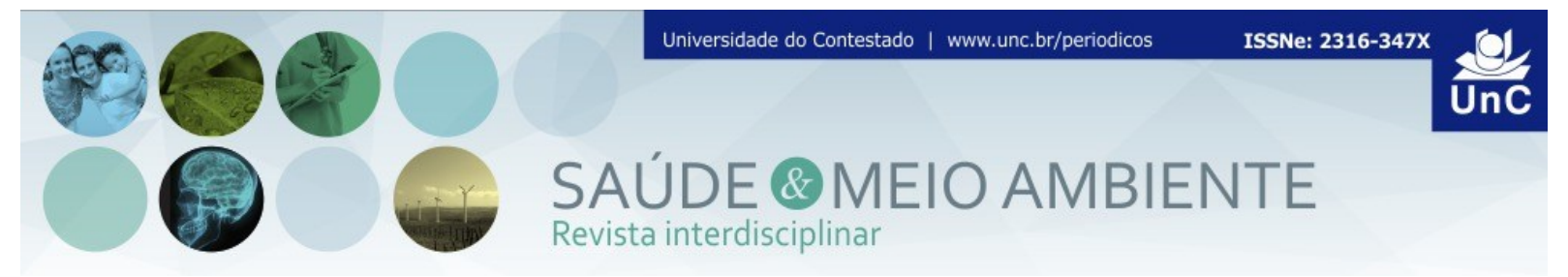

\title{
CONSUMO ALIMENTAR DE ADOLESCENTES DE DIFERENTES NÍVEIS DE ATIVIDADE FÍSICA
}

\author{
Leandro Smouter ${ }^{1}$ \\ André de Camargo Smolarek ${ }^{2}$ \\ Luis Paulo Gomes Mascarenhas ${ }^{3}$ \\ Tácito Pessoa de Souza Junior ${ }^{4}$
}

\begin{abstract}
RESUMO
Introdução: Elevadas taxas de alimentação inadequada e baixo nível de atividade física integram o estilo de vida do adolescente. Ademais, há necessidade de estudar esses fatores em cidades com menores dimensões geográficas, bem como analisalos em forma de contrastes, uma vez que, poucos estudos são encontrados na literatura. Objetivo: Comparar o consumo alimentar e o estado nutricional de adolescentes de diferentes níveis de atividade física. Metodologia: Participaram do estudo 76 adolescentes, com idade média de 12,76 $\pm 1,67$ anos, assim eles responderam questionários de atividade física e alimentação. Utilizou-se os testes U de Mann Whitney, teste $t$ independente e qui-quadrado com $\alpha$ de 0,05 para analisar os dados. Resultados: De oito grupos da pirâmide alimentar, um ajustou-se às recomendações nutricionais (carnes e ovos; $p=0,06$ ) com adequação de $123 \%$, quatro obtiveram consumo insuficiente (verduras e legumes $-86,7 \%$, frutas $-71 \%$, massas $-47,9 \%$ e leite e derivados $-30,7 \%$ ) e três revelaram consumo excessivo (feijões $+111 \%$, gorduras e óleos $+142 \%$ e açucares e doces $+521 \%$ ). Além disso, houve prevalência de adolescentes ativos $(83,8 \%)$ em relação aos pouco ativos $(16,2 \%)$. Conclusão: Não houve diferença do consumo alimentar e do estado nutricional entre adolescentes pouco ativos e ativos. Entretanto, são necessárias estratégias para aderência à alimentação mais qualificada, uma vez que, apenas um grupo alimentar esteve de acordo com as recomendações nutricionais.
\end{abstract}

Palavras-chave: Atividade Física. Educação Alimentar. Saúde do Adolescente.

\footnotetext{
${ }^{1}$ Acadêmico do curso de Educação Física da Universidade Estadual do Centro-Oeste (UNICENTRO). Irati. Paraná. Brasil. E-mail: leandrosmouter@hotmail.com

${ }^{2}$ Mestre em Educação Física pela Universidade Federal do Paraná (UFPR). Professor Colaborador do Departamento de Educação Física da Universidade Estadual do Centro-Oeste (UNICENTRO). Irati. Paraná. Brasil. E-mail: andrecsk@gmail.com

${ }^{3}$ Doutor em Saúde da Criança e do Adolescente pela Universidade Federal do Paraná (UFPR). Professor Titular do Departamento de Educação Física da Universidade Estadual do Centro-Oeste (UNICENTRO). Irati. Paraná. Brasil. E-mail: masca58@hotmail.com

${ }^{4}$ Doutor em Educação Física pela Universidade Estadual de Campinas (UNICAMP). Professor Adjunto III do Departamento de Educação Física da Universidade Federal do Paraná (UFPR). Curitiba. Paraná. Brasil. E-mail: tacitoir@ufpr.br
} 


\title{
FOOD CONSUMPTION OF ADOLESCENTS OF DIFFERENT LEVELS OF PHYSICAL ACTIVITY
}

\begin{abstract}
Introduction: The inadequate food intake and low physical activity level is integrate the teenager lifestyle. In addition, there is a need to study these factors in little cities, as well as analyzes them in form of contrasts, once, few studies are found in the literature. Objective: Compare food intake and nutritional status of teenagers of different levels of physical activity. Methodology: Participated in this study 76 subjects, with $12.76 \pm 1.67$ years old, like this they responded questionnaires of feed and physical activity. Was used tests $U$ of Mann Whitney, independent t-test and Chisquare with $\alpha$ of 0.05 to analyze the data. Results: Of eight groups of the food pyramid, one adjusted to the nutritional recommendations (meats and eggs; $p=0.06$ ) with $123 \%$ adequacy, four had insufficient consumption (vegetables $-86.7 \%,-71 \%$ fruits, pastas $-47.9 \%$ and milk and milk products $-30.7 \%$ ) and three revealed excessive consumptions (beans $+111 \%$, fats and oils $+142 \%$ and sugar and sweets $+521 \%$ ). Moreover, it occurred prevalence of active adolescents' (83.8\%) compared with little assets (16.2\%). Conclusion: No difference was showed of food consumption and nutritional status between two groups. However, strategies are required for adherence to the more qualified feeding, because only one food group fellow the nutritional recommendations.
\end{abstract}

Keywords: Physical Activity. Nutrition Education. Adolescent Health.

\section{INTRODUÇÃO}

O estilo de vida da sociedade contemporânea apresenta adversidades como elevadas taxas de alimentação inadequada e reduzido Nível de Atividade Física (NAF), ambas as adversidades são fatores de risco à saúde da população, sobretudo, destaca-se o público com faixa etária entre 10 e 19 anos, considerado adolescente pela Organização Mundial da Saúde (OMS), todavia, solucionar tais fatores de risco é um desafio para países desenvolvidos e em desenvolvimento (SCHWARTZ et al., 2013; EISENSTEIN, 2005).

Sabe-se que a inatividade física e a alimentação inadequada se relacionam com o aumento do número de sujeitos que desenvolvem patologias ligadas às Doenças Crônicas Não Transmissíveis (DCNT's), as quais comumente iniciam na adolescência e provocam maiores agravos à saúde na vida adulta (MONTEIRO et al., 2005; MOURA et al., 2008; CUNHA et al., 2008).

Diante do exposto, salienta-se que o debate sobre o assunto se intensificou no Brasil a partir do ano de 2004, durante a realização da segunda Conferência Nacional de Segurança Alimentar e Nutricional (CNSAN), a qual tinha como escopo 
o combate às DCNT's no país, este foi o momento em que se deliberou o Sistema Nacional de Segurança Alimentar e Nutricional (SISAN - Lei $\left.n^{\circ} 11.346 / 2006\right)$, uma das políticas fulcrais neste contexto (PINHEIRO, 2008; RECINE e VASCONCELLOS, 2011).

Sem dúvida o estabelecimento do SISAN foi um grande avanço para o país no sentido de ampliar a oferta e a possibilidade de aquisição de alimentos, contudo, é importante frisar que não basta apenas adquirir o alimento, pois nem todo tipo de alimento promove melhores condições de saúde ao indivíduo (MARTINEZ et al., 2013). A alimentação adequada integra à ingestão de nutrientes encontrados em diferentes grupos alimentares, assim entende-se que o hábito alimentar inadequado se traduz no grande número de calorias ingeridas e baixa demanda energética, o que reflete no balanço energético do indivíduo, pois a vasta ingestão calórica requer grande gasto energético, caso contrário, as taxas calóricas não utilizadas se convertem em tecido adiposo e, por conseguinte, desencadeia a obesidade (BOUCHARD, 2003).

A OMS recomenda por meio da Estratégia Global para a Promoção da Alimentação Saudável, Atividade Física e Saúde (EGPASAFS), que cada país formule e atualize periodicamente diretrizes nacionais voltadas para a orientação alimentar, as quais devem considerar os avanços científicos, culturais e regionais de cada país, o Brasil é adepto à recomendação da OMS e possui a diretriz disponível para toda a população no site do Ministério da Saúde (MS) (BRASIL, 2014).

Estudos sobre o tema apontam que o hábito alimentar de substituição de refeições importantes como café da manhã, almoço e jantar por lanches, salgados fritos, bolacha recheada e doces é comum entre adolescentes, um exemplo de tal hábito é descrito por Garcia, Gambardella e Frutuoso (2003) durante estudo efetuado com 153 adolescentes na cidade de São Paulo, no qual constataram que $70 \%$ dos adolescentes ingerem excessivamente alimentos de alta densidade energética. Carmo et al. (2006) encontraram quantidades elevadas de consumo de doces, sucos com adição de açúcar e refrigerantes no hábito alimentar de adolescentes, sendo que esse consumo substituía a ingestão do leite e de seus derivados.

De acordo com o MS, apenas $18,2 \%$ da população adolescente consome uma porção de frutas e hortaliças ao dia em pelo menos cinco dias semanais, quantidade suficiente para manter uma alimentação mais equilibrada, $34 \%$ consomem quantidades excessivas de alimentos com elevado teor de gordura e $28 \%$ consomem refrigerantes por mais de cinco vezes semanais, sendo que o consumo excessivo de gorduras e refrigerantes aumenta a incidência do excesso de peso corporal, refletindo em $62 \%$ da população atingida pela obesidade no país (BRASIL, 2011).

A obesidade é um segmento das DCNT's, que possuí fatores de risco modificáveis em comum, sendo que os principais são inatividade física e alimentação inadequada (BRASIL, 2011). A prática de atividade física nos níveis adequados, proporciona vários benefícios para a prevenção das DCNT's, dentre eles, a melhora do condicionamento muscular e cardiorrespiratório, aumento da 
saúde óssea e funcional, reduz o risco de hipertensão, patologia cardíaca coronária, acidente vascular cerebral (AVC) e diabetes do tipo II, além disso, a atividade física é fundamental para o balanço energético e controle de peso, independentemente da idade que o sujeito apresente (GUEDES et al., 2001; BOUCHARD, 2003).

A OMS define atividade física como sendo qualquer movimento corporal produzido pelos músculos esqueléticos que requeiram gasto energético acima da taxa metabólica basal, portanto, incluem atividades físicas praticadas durante o trabalho, jogos, execução de tarefas domésticas, viagens, atividades de lazer, exercício físico, dentre outras (LIMA; LEVY; LUIZ, 2014). Em estudo relacionado a fatores de risco para as DCNT's realizado pelo MS, constatou-se que o NAF da população brasileira se encontra abaixo do ideal, pois apenas $15 \%$ dos indivíduos praticam o suficiente para prevenir as DCNT's (BRASIL, 2011). Quanto a isso, à recomendação da OMS é de 60 minutos de atividade física diária de intensidade moderada à intensa para crianças e adolescentes de 5 a 18 anos de idade (ANTONY; BIDDLE; SALLIS, 2001).

Além disso, há necessidade de estudar esses temas em cidades com menores dimensões geográficas, uma vez que, poucos estudos são encontrados na literatura. Assim, o propósito deste estudo foi comparar o consumo alimentar e o estado nutricional de adolescentes conforme o NAF.

\section{MATERIAIS E MÉTODOS}

\section{DESENHO DO ESTUDO E PARTICIPANTES}

Trata-se de um estudo transversal do tipo ex-post-facto com amostragem por conveniência. Inicialmente foram convidados para participar do estudo 80 adolescentes, entretanto, foram considerados casos válidos apenas 76 , os quais responderam de forma correta os instrumentos da pesquisa, todos matriculados no ensino fundamental de um colégio público do município de Irati, PR. Além disso, foi solicitado a autorização do responsável legal por cada um dos participantes via Termo de Consentimento Livre e Esclarecido (TCLE). O estudo foi aprovado pelo Comitê de Ética em Pesquisa Envolvendo Seres Humanos (COMEP) da Universidade Estadual do Centro-Oeste (UNICENTRO) - parecer n 1372089/2015.

\section{COMPOSIÇÃO CORPORAL}

Para mesurar a massa corporal $(\mathrm{Kg})$ utilizou-se uma balança antropométrica digital da marca Líder $^{\circledR}$ com precisão de $100 \mathrm{~g}$ sob o seguinte protocolo: o avaliado 
em pé, de costas para a escala da balança, com afastamento lateral dos pés, olhar voltado para um ponto fixo e sem utilizar nenhum tipo de calçado (FILHO, 2003). A estatura corporal $(\mathrm{m})$ foi identificada através de uma fita métrica da marca Sanny ${ }^{\circledR}$ graduada em metros, centímetros e décimos de centímetros fixada na parede, sendo que o avaliado deveria estar na posição ortostática, sob apneia inspiratória, cabeça orientada segundo o plano de Frankfurt, usando roupa leve e estar sem qualquer tipo de calçado (FILHO, 2003). O índice de Massa Corporal (IMC) foi calculado através da formula $\left(\mathrm{kg} / \mathrm{m}^{2}\right)$, posteriormente, realizou-se o cálculo do score-z por meio do software AnthroPlus ${ }^{\circledR}$ da OMS, a classificação foi realizada através do score-z dentro de categorias de desvio padrão (DP) conforme Ferrari et al. (2015) da seguinte forma: $<-1 \mathrm{DP}=$ magreza, $\geq-1 \mathrm{DP}$ e $\leq+1 \mathrm{DP}=$ eutrófico, $>+1 \mathrm{DP}$ e $\leq+2$ $\mathrm{DP}=$ excesso de peso e $>+2 \mathrm{DP}=$ obesidade.

\section{NÍVEL DE ATIVIDADE FÍSICA}

Em relação ao NAF, utilizou-se o questionário Physical Activity Checklist Interview (PACl) na versão brasileira com adaptação transcultural validado em adolescentes por Cruciani et al. (2011), cujo título em idioma português é "Lista de Atividades Físicas (LAF). O PACl permite identificar o tempo dedicado para atividades físicas no dia anterior e possuí unidade de medida em minutos, sendo que são consideradas atividades com tempo $\geq 10$ minutos de duração, sendo 21 categorias de atividades pré-definidas como, por exemplo, andar de bicicleta, caminhada, esportes com raquete, dentre outras. As atividades praticadas pelo voluntário e que não constam nas categorias do questionário são inseridas em espaço reservado para outras atividades no mesmo questionário, assim também são contabilizadas. Além disso, as atividades são questionadas em quatro períodos: antes da escola, durante a escola, na aula de educação física e depois da escola. Por fim, soma-se o tempo dedicado às atividades que resulta no montante pelo qual se obtém o NAF.

\section{CONSUMO ALIMENTAR}

O hábito de consumo alimentar foi constatado por meio do Questionário de Frequência Alimentar da Pirâmide Alimentar (QFA-PA), adaptado para adolescentes e validado por Martinez (2013). O QFA-PA quantifica o consumo médio de porções alimentares de 8 grupos da pirâmide alimentar, são eles: massas, frutas, verduras e legumes, carnes e ovos, leite e derivados, feijão, gorduras e óleos e açucares e doces. Para isso, ele questiona o consumo de alimentos que se enquadram em cada um desses grupos, sendo que a unidade de medida adotada é o número de porções, as quais variam de acordo com o tipo de alimento, portanto, podem ser, colher, ponta de faca, copo, dentre outras. Inicialmente se constata a frequência de consumo diário, cujo enquadramento acontece em uma das seguintes categorias: 2 
ou mais vezes por dia $=2$ porções/dia, 1 vez por dia $=1$ porção/dia, 2 a 4 vezes $/$ semana $=0,43$ porções $/ \mathrm{dia}, 1 \mathrm{vez} / \mathrm{semana}=0,14 \mathrm{porções} / \mathrm{dia}, 1$ a 3 vezes $/ \mathrm{mês}=0,07$ porções $/ \mathrm{dia}$, menos de $1 \mathrm{vez} / \mathrm{mês}=0$ porções $/ \mathrm{dia}$ e nunca $=0$ porções/dia. Após identificar a frequência, se identifica o número de porções/vez, que é nada mais do que o número de porções ingeridas durante cada uma das frequências mencionadas anteriormente, assim resultando na seguinte equação: porções/dia $\times$ porções/vez $=$ consumo médio/dia. Esse procedimento é realizado para cada tipo de alimento, sendo que posteriormente somam-se os valores médios de todos os alimentos que compõem o grupo, assim retornando o consumo médio de cada grupo da pirâmide.

\section{ANÁLISE ESTATÍSTICA}

Os dados foram tabulados e analisados no software IBM ${ }^{\circledR}$ SPSS $^{\circledR}$ - Statistical Package for the Social Sciences - versão 21. Os procedimentos utilizados foram: estatística descritiva - frequência simples, frequência relativa, média e desvio padrão; estatística analítica - teste de normalidade de Kolmogorov-Smirnov, teste qui-quadrado de Pearson e teste não paramétrico $U$ de Mann-Whitney; percentual de adequação (\%) - [ (média de consumo $\div$ recomendação média) x100 ] com discrepância medida pelo teste $t$ de Student de uma amostra. Todas as análises adotaram $\alpha$ de 0,05 .

\section{RESULTADOS E DISCUSSÕES}

A tabela 1 evidencia a caracterização da amostra através das variáveis de medidas antropométricas, prática de atividade física e hábito alimentar, cujos valores referem-se à média e o desvio padrão de cada variável. Além disso, buscou-se a comparação das variáveis entre os sexos, entretanto, houve diferença significativa apenas nas variáveis estatura $(p=0,030)$ e minutos de atividade física no dia anterior $(p=0,028)$. 
Tabela 1 - Caracterização da amostra com diferença entre os sexos.

\begin{tabular}{ccccc}
\hline \multirow{2}{*}{ Variável } & $\begin{array}{c}\text { Total } \\
\mathbf{7 6 ( 1 0 0 \% )}\end{array}$ & $\begin{array}{c}\text { Masculino } \\
\mathbf{4 5}(\mathbf{5 9 , 2} \%)\end{array}$ & $\begin{array}{c}\text { Feminino } \\
\mathbf{3 1}(\mathbf{4 0 , 8} \%)\end{array}$ & \multirow{2}{*}{$\mathbf{p}^{* *}$} \\
\cline { 2 - 4 } & Média (DP) & Média (DP) & Média (DP) & \\
\hline Idade (anos) & $12,76 \pm 1,67$ & $12,98 \pm 1,69$ & $12,45 \pm 1,60$ & 0,129 \\
MC (Kg) & $51,57 \pm 15,77$ & $53,82 \pm 17,47$ & $48,29 \pm 12,48$ & 0,197 \\
Estatura (m) & $1,59 \pm 0,12$ & $1,61 \pm 0,14$ & $1,55 \pm 0,08^{*}$ & 0,030 \\
IMC & $19,98 \pm 3,61$ & $20,10 \pm 3,46$ & $19,81 \pm 3,87$ & 0,485 \\
AF (min./dia) & $198 \pm 140$ & $224 \pm 143$ & $161 \pm 129^{*}$ & 0,028 \\
Massas (P) & $4,69 \pm 2,34$ & $4,93 \pm 2,51$ & $4,34 \pm 2,04$ & 0,308 \\
Frutas (P) & $1,45 \pm 1,51$ & $1,37 \pm 1,33$ & $1,62 \pm 1,58$ & 0,526 \\
VL (P) & $0,53 \pm 0,51$ & $0,49 \pm 0,47$ & $0,60 \pm 0,57$ & 0,557 \\
CO (P) & $2,46 \pm 2,14$ & $2,66 \pm 2,30$ & $2,18 \pm 1,90$ & 0,347 \\
LD (P) & $2,77 \pm 2,21$ & $2,78 \pm 2,41$ & $2,75 \pm 1,93$ & 0,857 \\
Feijão (P) & $2,11 \pm 1,79$ & $2,43 \pm 2,10$ & $1,65 \pm 1,09$ & 0,165 \\
GÓ (P) & $2,42 \pm 2,13$ & $2,07 \pm 2,04$ & $2,92 \pm 2,19$ & 0,080 \\
AD (P) & $6,21 \pm 4,50$ & $5,84 \pm 4,11$ & $6,74 \pm 5,04$ & 0,586 \\
\hline
\end{tabular}

Notas: $\mathrm{DP}=$ desvio padrão; ${ }^{* *}=p$-valor do teste $U$ de Mann-Whitney; ${ }^{*}=$ diferença significativa entre os

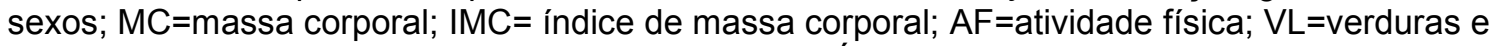

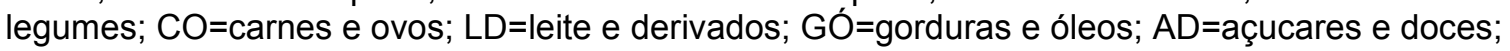
$\mathrm{P}=$ porções.

Mesmo com distribuição bastante dispersa da variável atividade física observada por meio dos desvios padrões próximos da média, ainda é possível afirmar com uma probabilidade de erro de $2,8 \%$ que os meninos são mais ativos que as meninas. Essa distribuição pode ser melhor compreendida por meio do gráfico 1 , no qual observa-se que o maior tempo de atividade física apontado é do sexo feminino, localizado entre 650 e 700 minutos, já do sexo masculino, a maior marca está entre 600 e 650 minutos. Entretanto, o menor índice é próximo entre os sexos, localizando-se entre 10 e 50 minutos diários. Portanto, o que caracteriza a diferença média entre os sexos é a distribuição do tempo desigual nos scores centrais. 
Gráfico 1 - Tempo de prática de atividade física no dia anterior conforme o sexo.

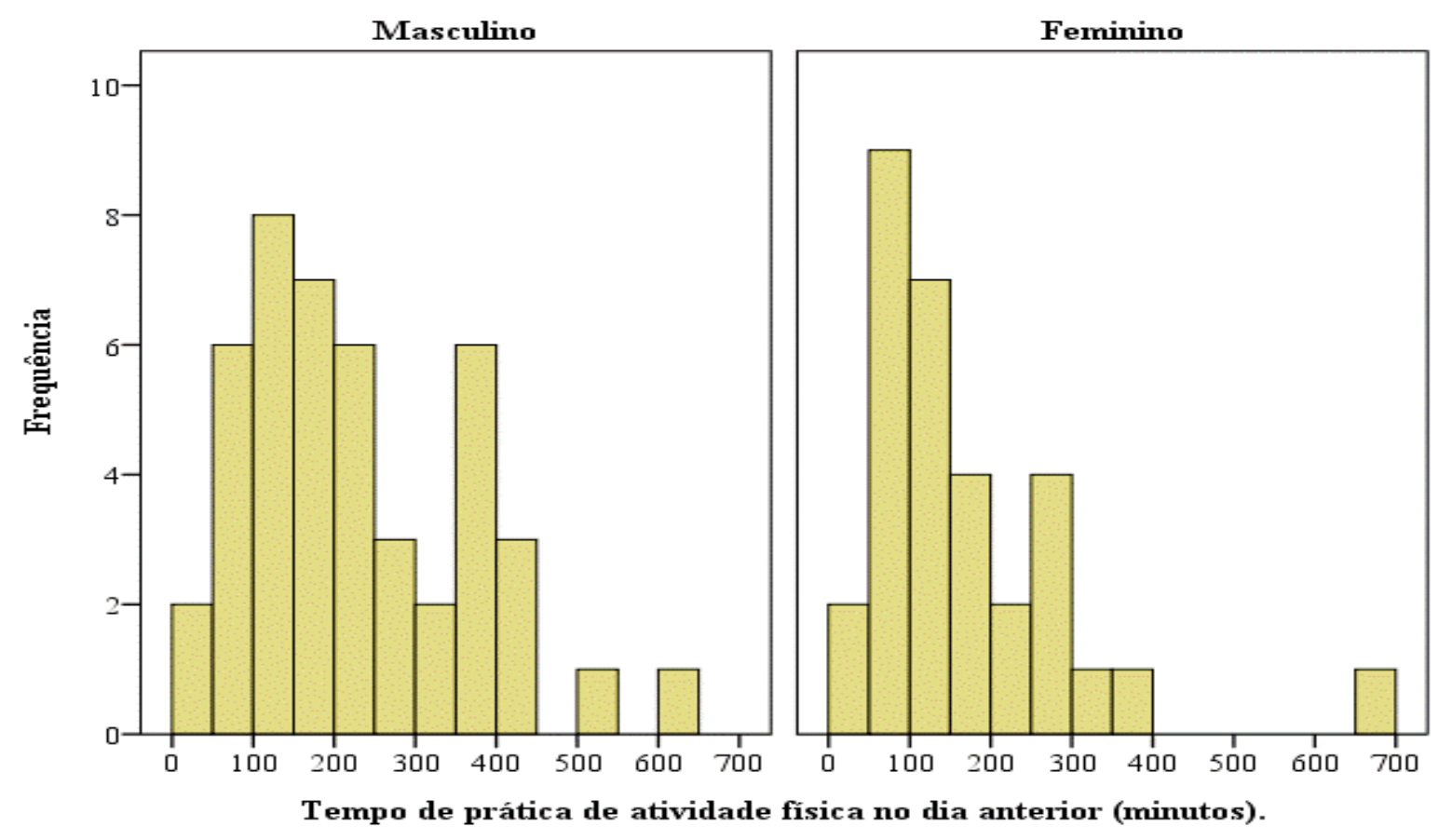

Em relação à categorização no NAF, adotou-se a recomendação da OMS, cujo tempo de prática para adolescentes é de no mínimo 60 minutos diários (ANTONY; BIDDLE; SALLIS, 2001). Desse modo, os voluntários da pesquisa foram classificados em dois grupos: pouco ativo e ativo. O grupo pouco ativo abrange os sujeitos que não atingem 60 minutos de prática de atividade física diária e o grupo ativo contempla os adolescentes que praticam 60 minutos ou mais de atividade física diariamente (Gráfico 2). 
Gráfico 2 - Distribuição de frequências simples e relativas do NAF.

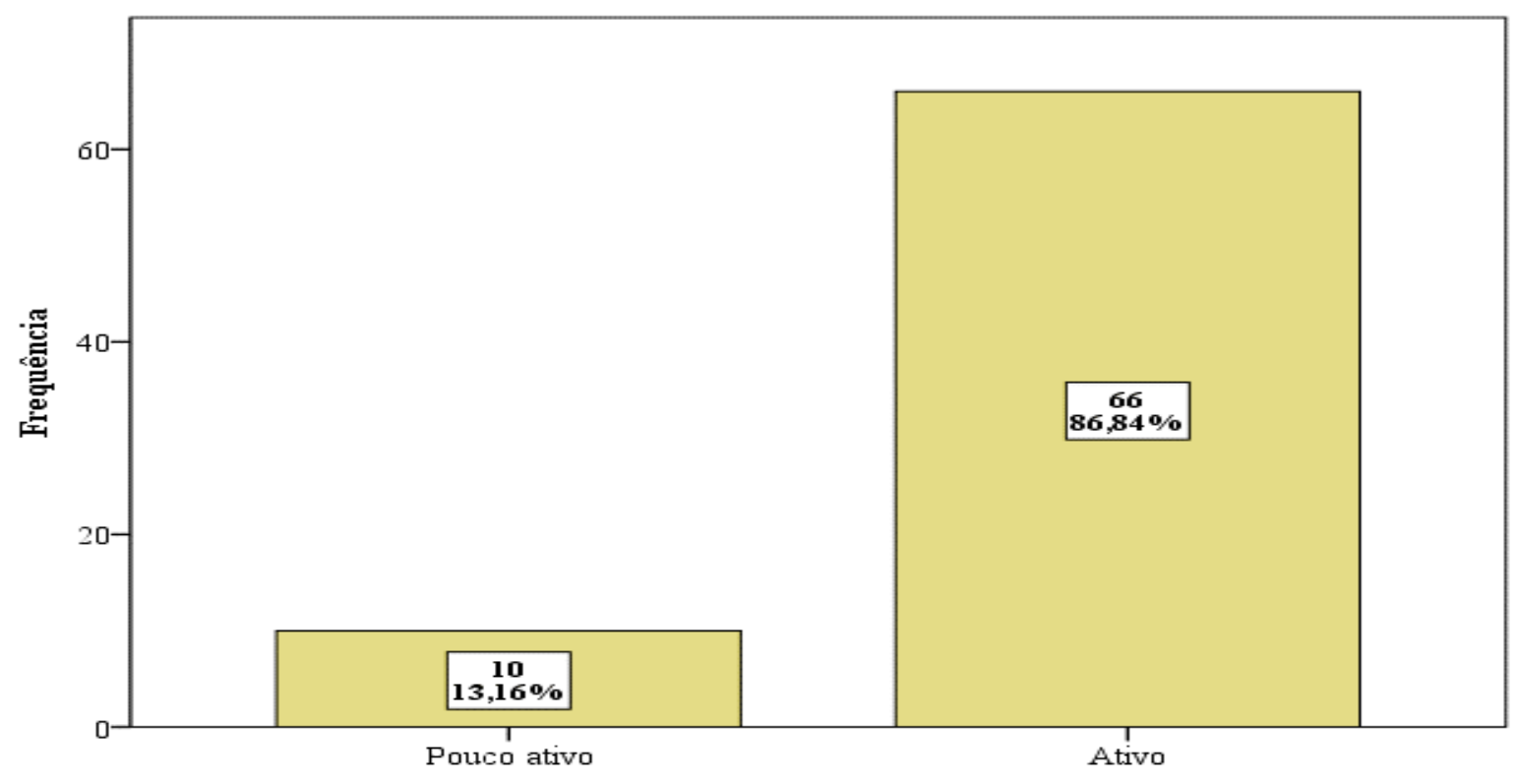

Nível de atividade física.

Houve prevalência de adolescentes ativos (86,84\%) em relação aos pouco ativos (13,16\%). Considerando a região geográfica do estudo, vamos de encontro a pesquisa realizado por Malta et al. (2014), na qual encontraram menos óbitos relacionados com às DCNT's na região sul do país, assim este estudo pode contribuir de maneira implícita na construção de elementos que giram em torno da conclusão de que onde há menos problemas de saúde relacionados às DCNT's, também há mais sujeitos ativos fisicamente.

A tabela 2 caracteriza o percentual de adequação do consumo médio de porções de alimentos dos participantes às Recomendações da Pirâmide Alimentar do Adolescente (RPAA).

Tabela 2 - Adequação do consumo médio de porções por grupo alimentar às RPAA.

\begin{tabular}{ccccccc}
\hline \multirow{2}{*}{ GA } & RPAA & Consumo & \multirow{2}{*}{ IC (95\%) } & DM & \multirow{2}{*}{$\%$ adequado } & $\mathbf{p}^{* *}$ \\
\cline { 2 - 3 } & Média & Média (DP) & & & & \\
\hline Massas (P) & 9 & $4,69 \pm 2,34^{*}$ & $4,17-5,22$ & $-4,30$ & $52,1 \%$ & 0,001 \\
Frutas (P) & 5 & $1,47 \pm 1,43^{*}$ & $1,15-1,80$ & $-3,52$ & $29 \%$ & 0,001 \\
VL (P) & 4 & $0,53 \pm 0,51^{*}$ & $0,42-0,65$ & $-3,46$ & $13,3 \%$ & 0,001 \\
CO (P) & 2 & $2,46 \pm 2,14$ & $1,98-2,95$ & 0,46 & $123 \%$ & 0,062 \\
LD (P) & 4 & $2,77 \pm 2,21^{*}$ & $2,27-3,27$ & $-1,22$ & $69,3 \%$ & 0,001 \\
Feijão (P) & 1 & $2,11 \pm 1,79^{*}$ & $1,71-2,53$ & 1,11 & $211 \%$ & 0,001 \\
GÓ (P) & 1 & $2,42 \pm 2,13^{*}$ & $1,94-2,90$ & 1,42 & $242 \%$ & 0,001 \\
AD (P) & 1 & $6,21 \pm 4,50^{*}$ & $5,20-7,23$ & 5,21 & $621 \%$ & 0,001 \\
\hline
\end{tabular}

Notas: GA=grupo alimentar; RPAA=recomendação média da pirâmide alimentar do adolescente; $\mathrm{DP}=$ desvio padrão; $\mathrm{IC}=$ intervalo de confiança para a média de consumo; $\mathrm{DM}=$ diferença média; $\%=$ percentual de adequação [ (média de consumo/recomendação) $\times 100$ ]; ${ }^{* *}=p$-valor do teste $t$ de Student para uma amostra; *=diferente significativa da recomendação; $P=$ =porções; VL=verduras e

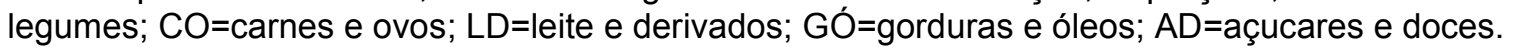


Constatou-se o consumo adequando em apenas um dos grupos alimentares, o grupo das carnes e ovos com percentual próximo ao adequado, já que se diferiu em apenas $23 \%$. Além disso, quatro grupos apresentaram consumo insuficiente: grupo de verduras e legumes com déficit de $86,7 \%$, grupo de frutas com déficit de $71 \%$, grupo de massas com déficit de $47,9 \%$ e o grupo de leite e derivados com déficit de $30,7 \%$. Já os três grupos restantes apresentaram consumo excessivo: grupo dos feijões com excesso de $111 \%$, grupo de gorduras e óleos com excesso de $142 \%$, além do grupo de açucares e doces com excesso de $521 \%$.

Com base nesses resultados, cabe destaque aos grupos alimentares que demonstraram elevada percentagem de excesso, ou seja, os grupos de gorduras e óleos e açucares e doces, sendo que ambos são considerados alimentos energéticos extras, altamente prejudiciais à saúde quando consumidos em excesso (MARTINEZ et al., 2013). Malta et al. (2014) explorando dados da OMS no ano de 2014, extraíram a conclusão de que o diabetes tipo II localiza-se entre as dez causas de morte no mundo, todavia, sabe-se que o diabetes tipo II tem primícias relacionadas ao elevado consumo de açucares, inclusive, enquadrado como um dos principais fatores de risco para o diabetes tipo II. Entretanto, a amostra do presente estudo demonstrou manter o hábito de consumir numerosos índices deste componente alimentar (Tabela 2).

Nessa linha de estudo, é necessário retomar o diálogo com Malta et al. (2014), já que também explanaram elevado consumo de açucares na alimentação de adolescentes, entretanto, o percentual encontrado não chegou nem à $50 \%$ do excesso encontrado neste estudo. Além disso, às chances de um sujeito que consome elevados índices de açucares na fase da adolescência manter esse comportamento irregular na idade adulta é maior do que um sujeito que zela pelo equilíbrio nutricional (MARTINEZ et al., 2013). Portanto, o esforço para reduzir os índices de prevalência do diabetes tipo II em adultos deve ser iniciado já na adolescência para que germinem melhores resultados. Desse modo, o estudo tornase importante para que intervenções nesse sentido sejam projetadas para essa população.

Carmo et al. (2006) também constataram elevado índice do consumo de porções de açúcares pelos adolescentes do município de Piracicaba, SP, cujo valor calculado foi de 3,8 porções diárias, entretanto, houve menor consumo do que o constatado neste estudo (Tabela 2). Todavia, salienta-se que o município de Piracicaba possui maior densidade demográfica $\left(264,47\right.$ habitantes $\left./ \mathrm{km}^{2}\right)$ do que o município de Irati $\left(56,23\right.$ habitantes $\left./ \mathrm{km}^{2}\right)$, fato que pode contribuir uma circulação mais profícua de informações relacionadas ao consumo alimentar consciente e, por conseguinte, maior clareza dos adolescentes sobre as recomendações propostas em políticas deste cunho como, por exemplo, as que foram discutidas na introdução deste estudo.

Schwartz et al. (2013) estudaram o perfil nutricional de adolescentes do município de Caiobá, MT, para concluir que o controle alimentar provoca alterações importantes no estado nutricional deste público. Além disso, sabe-se que para o referido controle ser favorável para ao organismo, é necessário que ele se aproxime 
das recomendações, além de haver um equilíbrio entre consumo alimentar e gasto energético.

Nesse sentido, a tabela 3 representa a comparação do consumo médio por Grupo da Pirâmide Alimentar do Adolescente (GPAA) entre as duas categorias do NAF: grupo pouco ativo (GPA) e grupo ativo (GA).

Tabela 3 - Comparação do consumo alimentar entre GPA e GA.

\begin{tabular}{ccccccc}
\hline \multirow{2}{*}{ GPAA } & \multicolumn{2}{c}{ GPA } & & \multicolumn{2}{c}{ GA } & \multirow{2}{*}{ * $^{*}$} \\
\cline { 2 - 3 } \cline { 6 - 7 } & Média (DP) & $\%$ & & Média (DP) & $\%$ & \\
\hline Massas (P) & $3,82 \pm 1,31$ & $42,5 \%$ & & $4,83 \pm 2,43$ & $53,7 \%$ & 0,373 \\
Frutas (P) & $0,78 \pm 0,53$ & $15,7 \%$ & & $1,57 \pm 1,50$ & $31,6 \%$ & 0,195 \\
Verdura e legume (P) & $0,26 \pm 0,13$ & $6,5 \%$ & & $0,57 \pm 0,54$ & $14,4 \%$ & 0,181 \\
Carnes e ovos (P) & $2,90 \pm 3,13$ & $145,2 \%$ & & $2,40 \pm 1,98$ & $120,1 \%$ & 0,920 \\
Leite e derivados (P) & $2,51 \pm 1,19$ & $62,8 \%$ & & $2,81 \pm 2,33$ & $70,3 \%$ & 1,000 \\
Feijão (P) & $1,21 \pm 0,76$ & $121,4 \%$ & & $2,25 \pm 1,86$ & $225,3 \%$ & 0,074 \\
Gorduras e óleos (P) & $1,54 \pm 1,61$ & $154,8 \%$ & & $2,55 \pm 2,18$ & $255,4 \%$ & 0,180 \\
Açucares e doces (P) & $5,15 \pm 4,86$ & $515,1 \%$ & & $6,37 \pm 4,46$ & $637,6 \%$ & 0,255 \\
\hline
\end{tabular}

Notas: GPAA=grupo da pirâmide alimentar do adolescente; GPA=grupo pouco ativo; GA=grupo ativo; $\mathrm{DP}=$ desvio padrão; $\mathrm{P}=$ porções; \%=percentual de adequação às recomendações da pirâmide alimentar do adolescente; * $=p$-valor do teste $U$ de Mann-Whitney.

Não houve diferença do consumo médio dos grupos alimentares entre as duas categorias do NAF. Entretanto, ao equacionar o desfecho de não haver diferença entre o consumo alimentar dos adolescentes pouco ativos e ativos, resulta num desdobramento insatisfatório, pois à medida que os adolescentes de ambos os grupos ingerem igualmente elevadas taxas de alimentos energéticos como massas e energéticos extras como açucares e doces, porém realizam atividade física de forma desigual, subentende-se que os adolescentes pouco ativos terão maiores chances de desenvolver DCNT's, já que o balanço energético encontrar-se-á em desiquilíbrio. 
Gráfico 3 - Classificação do IMC conforme o NAF.

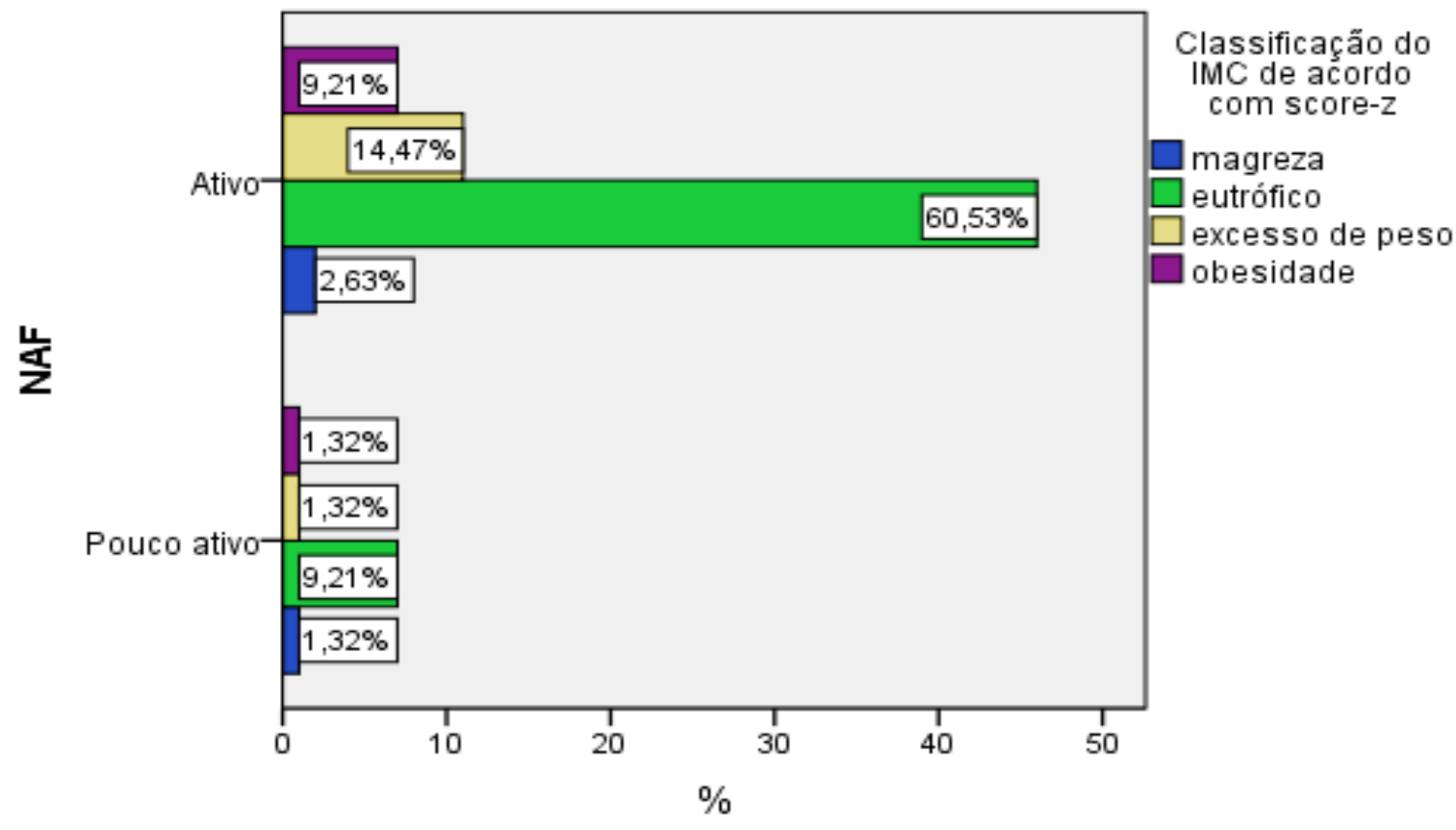

Diante do exposto, o gráfico 3 exemplifica o estado nutricional dos adolescentes segundo a classificação do NAF, porém não houve diferença significativa entre as proporções $\left(x^{2}=1,31 ; p=0,725\right)$. Todavia, destaca-se a grande prevalência de adolescentes ativos classificados como eutróficos $(60,53 \%)$ em relação aos pouco ativos $(9,21 \%)$.

Fonseca e Corbo (2007) defendem um preâmbulo importante sobre os temas discutidos neste manuscrito, trata-se da equalização da responsabilidade de manter hábitos alimentares satisfatórios para a promoção da saúde e qualidade de vida entre o sujeito e o estado, que em outras palavras, significa dizer que o sujeito não pode receber toda a culpa por não conseguir manter hábitos que são considerados adequados para a saúde, pois isso também depende do estado que interage no sentido de assegurar condições para isso através de políticas públicas deste cunho.

Contudo, há de se entender que ao analisar a adequação do consumo às recomendações da pirâmide do adolescente (Tabela 2), os resultados não foram satisfatórios, porém o NAF demonstrou maior prevalência de adolescentes ativos (Gráfico 2). Desse modo, é possível conversar sobre a eficácia das políticas públicas levantadas inicialmente pelo estudo, cujo às que são voltadas para a conscientização da prática de atividade física parecem surtir maiores efeitos do que às voltadas para orientar o consumo alimentar em cidades com menores expressões geográficas como é o caso da Irati.

Além disso, Fonseca e Corbo (2007) apontam para controvérsias num dos pressupostos das políticas públicas voltadas para orientar condutas que propiciam melhores condições de saúde, cujo a titularidade desse pressuposto é o princípio da equidade, o qual em sua versão controversa denomina-se de iniquidade. Os autores 
realçam esse termo porque o entendem como determinante para as condutas pessoais relacionadas à saúde. Em outras palavras, entende-se que o sujeito precisa de condições mais próximas dele para que seja possível adotar hábitos mais plausíveis do ponto de vista da saúde satisfatória.

Nessa linha de estudo, dois exemplos conexos aos resultados desta pesquisa podem ser representados de forma extremamente simples: primeiro - houve grande prevalência de adolescentes ativos e segundo - houve elevados índices de consumo de gorduras e óleos entre os adolescentes porque seus hábitos são inadequados. Esses dois exemplos poderiam estar esgotados nessas conclusões, porém sob um olhar mais reduzido do conceito de saúde, assim fortalecendo a iniquidade. Entretanto, sob o olhar de equidade apontado por Fonseca e Corbo (2007), se buscaria responder de outra forma esses dois exemplos, sendo que uma forma de fazer isto seria: primeiro - houve prevalência de adolescentes ativos porque o ambiente físico possibilitou isso à eles e segundo - houve elevados índices de consumo de gorduras e óleos porque as condições econômicas das famílias destes adolescentes não possibilitaram a aquisição de alimentos com menores percentuais de gorduras e óleos, ou seja, não basta apenas orientar os adolescentes, mas também criar possibilidades de efetivação das orientação passadas.

Pinheiro (2008) estudando o desenvolvimento histórico e político da Lei Orgânica de Segurança Alimentar (SAN) no Brasil, também enfatiza que o significado do direito da população ao acesso periódico e contínuo aos alimentos de qualidade sob os níveis suficientes para o equilíbrio nutricional não são bem entendido no processo de implementação das políticas, assim o autor afirma que ainda há barreiras que precisam ser superadas, mas que infelizmente essa controvérsia ainda é uma lacuna nas políticas deste cunho.

Sem mais, também se menciona que o estudo apresenta limitações, primeiro, uma parcela de fragilidade no instrumento $\mathrm{PACl}$ quando detectou índices de atividade física incomuns ao ser humano (muito acima do normal), embora tenha sido apenas dois casos e, segundo, o estudo possuir baixa representatividade amostral.

\section{CONCLUSÃO}

Conclui-se que não houve diferença significativa do consumo alimentar entre adolescentes pouco ativos e ativos, bem como, do estado nutricional conforme o NAF. Entretanto, são necessárias estratégias para aderência à alimentação mais qualificada, uma vez que, apenas um grupo alimentar esteve de acordo com as recomendações nutricionais. 


\section{REFERÊNCIAS}

ANTONY, N. C.; BIDDLE, S. J. H.; SALLIS, J. F. Health enhancing physical activity for young people: statement of United Kingdom expert consensus conference. Pediatric exercise science, Ontário, Canadá, v. 13, n. 1, p. 12-25, fev. 2001.

BOUCHARD, C. Atividade física e obesidade. São Paulo: Manole, 2003.

BRASIL. Plano de ações estratégicas para o enfrentamento das doenças crônicas não transmissíveis (DCNT) no Brasil 2011 - 2022. 1. ed. Brasília: Ministério da Saúde, 2011.

BRASIL. Guia alimentar para a população brasileira. 2. ed. Brasília: Ministério da Saúde, 2014.

CARMO, M. B. D. et al. Consumo de doces, refrigerantes e bebidas com adição de açúcar entre adolescentes da rede pública de ensino de Piracicaba, São Paulo.

Revista Brasileira de Epidemiologia, São Paulo, v. 9, n. 1, p. 121-130, mar. 2006.

CRUCIANI, F. et al. Equivalência conceitual, de itens e semântica do Physical Activity Checklist Interview (PACl). Cadernos de Saúde Pública, Rio de Janeiro, v. 27, n. 1, p. 19-34, jan. 2011.

CUNHA, I. C. et al. Fatores associados à prática de atividade física na população adulta de Goiânia: monitoramento por meio de entrevistas telefônicas. Revista Brasileira de Epidemiologia, São Paulo, v. 11, n. 3, p. 495-504, set. 2008.

EISENSTEIN, E. Adolescência: definições, conceitos e critérios. Adolescência e saúde, Rio de Janeiro, v. 2, n. 2, p. 6-7, jun. 2005.

FERRARI, G. L. D. M. et al. Associação entre equipamentos eletrônicos no quarto com tempo sedentário, atividade física e índice de massa corporal de crianças.

Revista de Pediátria, Rio de Janeiro, v. 91, n. 6, p. 574-582, nov./dez. 2015.

FILHO, J. F. A prática da avaliação física: teste, medidas e avaliação física em escolares, atletas e academias de ginástica. 2. ed. Rio de Janeiro: Shape, 2003.

FONSECA, A. F.; CORBO, A. D. O território e o processo saúde-doença. Rio de Janeiro: EPSJV/Fiocruz, 2007.

GARCIA, G. C. B.; GAMBARDELLA, A. M. D.; FRUTUOSO, M. F. P. Estado nutricional e consumo alimentar de adolescentes de um centro de juventude da cidade de São Paulo. Revista de Nutrição, Campinas, v. 16, n. 1, p. 41-50, jan./mar. 2003.

GUEDES, D. P. et al. Níveis de prática de atividade física habitual em adolescentes. Revista Brasileira de Medicina do Esporte, Niterói, v. 7, n. 6, p. 187-199, nov./dez. 2001. 
LIMA, D. F. D.; LEVY, R. B.; LUIZ, O. D. C. Recomendações para atividade física e saúde: consensos, controvérsias e ambiguidades. Revista Panamericana de Salud Pública, Washington, EUA, v. 36, n. 3, p. 164-170, set. 2014.

MALTA, D. C. et al. Mortalidade por doenças crônicas não transmissíveis no Brasil e suas regiões, 2000 a 2011. Epidemiologia e Serviços de Saúde, Brasília, v. 23, n. 4, p. 599-608, dez. 2014.

MARTINEZ, M. F. et al. Validade e reprodutibilidade de um questionário de frequência alimentar para avaliar a ingestão grupo de alimentos em adolescentes. Cadernos de Saúde Pública, Rio de Janeiro, v. 29, n. 9, p. 1795-1804, set. 2013.

MONTEIRO, C. A. et al. Monitoramento de fatores de risco para doenças crônicas por entrevistas telefônicas. Revista de Saúde Pública, São Paulo, v. 39, n. 1, p. 4757, jan. 2005.

MOURA, E. C. et al. Vigilância de Fatores de Risco para Doenças Crônicas por Inquérito Telefônico nas capitais dos 26 estados brasileiros e no Distrito Federal (2006). Revista Brasileira de Epidemiologia, São Paulo, v. 11, n. 1, p. 20-37, suppl. 2008.

PINHEIRO, A. R. D. O. Reflexões sobre o processo histórico/político de construção de Lei Orgânica de Segurança Alimentar e Nutricional. Segurança Alimentar e Nutricional, Campinas, v. 15, n. 2, p. 1-15, jul. 2008.

RECINE, E.; VASCONCELLOS, A. B. Políticas nacionais e o campo da Alimentação e Nutrição em Saúde Coletiva: cenário atual. Ciência e Saúde Coletiva, Rio de Janeiro, v. 16, n. 1, p. 73-79, jan. 2011.

SCHWARTZ, A. J. et al. Nível de atividade física e estado nutricional em escolares do ensino fundamental. Revista Brasileira de Obesidade, Nutrição e

Emagrecimento, São Paulo, v. 7, n. 40, p. 84-87, jul./ago. 2013.

Artigo recebido em: 24/11/2016

Artigo aprovado em: 27/04/2017 